\title{
Bone marrow concentrate-induced mesenchymal stem cell conditioned medium facilitates wound healing and prevents hypertrophic scar formation in a rabbit ear model
}

Ching-Hsuan Hu ${ }^{1,2,3}$, Yi-Wen Tseng ${ }^{1,2,3}$, Chih-Yung Chiou², Kuan-Chun Lan ${ }^{4}$, Chih-Hung Chou ${ }^{5}$, Chun-San Tai ${ }^{6}$, Hsien-Da Huang ${ }^{7}$, Chiung-Wen Hü ${ }^{8}$, Ko-Hsun Liao ${ }^{2}$, Shiow-Shuh Chuang ${ }^{1}$, Jui-Yung Yang ${ }^{1}$ and Oscar K. Lee ${ }^{2,3^{*}}$

\begin{abstract}
Background: Hypertrophic scars (HSs) are formed via an aberrant response to the wound healing process. HSs can be cosmetic or can result in functional problems. Prolonged proliferation and remodeling phases disrupt wound healing, leading to excessive collagen production and HS formation. However, there are currently no satisfactory drugs to prevent HS formation. Mesenchymal stem cell (MSC) conditioned medium (CM) has therapeutic effects on wound healing and preventing HS formation. Bone marrow concentrate (BMC) contains various growth factors and cytokines that are crucial for regeneration and has been applied in the clinical setting. In this study, we evaluated the effects of BMC-induced MSC CM on HS formation in a rabbit ear model.

Methods: We established a rabbit ear wound model by generating full-thickness wounds in the ears of rabbits $(n=12)$ and treated wounds with MSC CM, BMC CM, or BMC-induced MSC CM. Dermal fibroblasts from human hypertrophic scar were stimulated with transforming growth factor beta 1 (TGF- $\beta 1$ ) for $24 \mathrm{~h}$ and cultured in each culture medium for $72 \mathrm{~h}$. We measured the hypertrophic scar (HS) formation during the skin regeneration by measuring the expression of several remodeling molecules and the effect of these conditioned media on active human HS fibroblasts.

Results: Our results showed that BMC-induced MSC CM had greater antifibrotic effects than MSC CM and BMC CM significantly attenuated HS formation in rabbits. BMC-induced MSC CM accelerated wound re-epithelization by increasing cell proliferation. Additionally, BMC-induced MSC CM also inhibited fibrosis by decreasing profibrotic gene and protein expression, promoting extracellular matrix turnover, inhibiting fibroblast contraction, and reversing myofibroblast activation.
\end{abstract}

Conclusions: BMC-induced MSC CM modulated the proliferation and remodeling phases of wound healing, representing a potential wound healing agent and approach for preventing HS formation.

Keywords: Hypertrophic scar, Bone marrow concentrate, Mesenchymal stem cell-conditioned medium

\footnotetext{
* Correspondence: oscar.lee@cuhk.edu.hk

${ }^{2}$ Institute of Clinical Medicine, National Yang-Ming University, Taipei, Taiwan

${ }^{3}$ Stem Cell Research Center, National Yang-Ming University, Taipei, Taiwan

Full list of author information is available at the end of the article
}

(c) The Author(s). 2019 Open Access This article is distributed under the terms of the Creative Commons Attribution 4.0 International License (http://creativecommons.org/licenses/by/4.0/), which permits unrestricted use, distribution, and reproduction in any medium, provided you give appropriate credit to the original author(s) and the source, provide a link to the Creative Commons license, and indicate if changes were made. The Creative Commons Public Domain Dedication waiver (http://creativecommons.org/publicdomain/zero/1.0/) applies to the data made available in this article, unless otherwise stated. 


\section{Introduction}

Hypertrophic scars (HSs) are a common complication of burns and other soft tissue injuries. After injury to dermal tissues, HSs can occur owing to fibroblast proliferation, inflammatory cell infiltration, and abnormal extracellular matrix (ECM) accumulation and remodeling, particularly with regard to collagen [1]. Several treatments for HSs, including surgical excision, intralesional corticosteroid injection, compression, laser treatment, and interferon injection, have been developed to date [1]. However, these treatments are not effective for preventing excessive scar tissue formation and regenerating healthy tissue. Hence, management of HS formation remains a challenge.

Bone marrow concentrate (BMC) has been used extensively in regenerative medicine [2]. BMC is an autologous bone marrow-derived product that provides a heterogeneous mixture of cells and a variety of bioactive growth factors and cytokines associated with wound healing [3]. BMC is also easy to collect, enabling immediate treatment of injuries [3]. BMC has been applied in patients with ischemic wounds, osteoporosis, arthritis, and tendon healing, yielding promising outcomes [2-6]. Although the specific mechanisms through which BMC affects wound healing have not yet been well established, several mechanisms have been proposed, including increasing tissue vascularity and tissue microperfusion at the capillary level, remodeling of fibrotic tissues to allow new capillary growth or to increase interstitial fluid flow, and modulating the inflammatory response [5].

MSCs are multipotent with self-renewal and multiple differentiation capacities. MSCs are promising in a variety of antifibrosis applications owing to their effects on attenuating collagen deposition [7]. Paracrine mechanisms mediate the therapeutic effects of MSCs, and trophic factors secreted from MSCs suppress inflammation and apoptosis, but promote angiogenesis and mitosis in parenchymal cells [8]. MSCs and BMC are effective for accelerating wound healing. However, it is unclear whether BMC can promote the therapeutic effects of MSCs on wound healing and HS formation. The bioactive cytokines in BMC may enhance MSC regenerative capacity to prevent HS formation.

Accordingly, in this study, we aimed to validate the therapeutic effects of conditioned medium $(\mathrm{CM})$ from BMC-induced MSCs on HSs in a rabbit model.

\section{Materials and methods}

\section{Patients and tissue samples}

All HS tissues were harvested at the time of surgery from four patients confirmed to have clinical and pathological evidence of HSs and who had not yet received scar treatment. Before surgery, all patients provided verbal and written consent to take part in the study. The study was approved by the Medical and Ethics Committee of our hospital (Institutional Review Board approval number: 201701193B0).

\section{Isolation and culture of scar-derived fibroblasts}

Dermal fibroblast cultures were established from tissue specimens, which were processed within $4 \mathrm{~h}$ after surgical excision, as previously described [9]. Briefly, the epidermis of specimens was removed, and the dermis was minced into small pieces $\left(1 \mathrm{~mm}^{3}\right)$. The dermis was repeatedly washed in sterile Dulbecco's modified Eagle's medium (DMEM; Gibco, NY, USA) supplemented with $100 \mathrm{U} / \mathrm{mL}$ penicillin, $100 \mathrm{mg} / \mathrm{mL}$ streptomycin, and $10 \%$ fetal bovine serum (FBS; Gibco). The specimens were then exposed to $10 \mathrm{mg} / \mathrm{mL}$ collagenase type IV (SigmaAldrich, MO, USA) and $10 \mathrm{mg} / \mathrm{mL}$ Dispase II (Invitrogen, CA, USA) in DMEM and incubated at $37^{\circ} \mathrm{C}$ in a $5 \%$ $\mathrm{CO}_{2}$ atmosphere for $3 \mathrm{~h}$. After enzymatic digestion, the cell suspension was filtered through a $70-\mu \mathrm{m}$ cell strainer (BD Biosciences, CA, USA) and centrifuged at $200 \times g$ for $4 \mathrm{~min}$. The supernatant was discarded, and the cell pellet was resuspended in sterile DMEM (Gibco) supplemented with $100 \mathrm{U} / \mathrm{mL}$ penicillin, $100 \mathrm{mg} / \mathrm{mL}$ streptomycin, and 10\% FBS (Gibco). The cells were cultured in 100-mm plastic tissue culture dishes (BD Biosciences). Once confluent scar-derived fibroblasts were established as monolayer cultures, cell passaging was performed using $0.125 \%$ trypsin (Gibco). Scar-derived fibroblasts between the sixth and 11th passages were used for experiments.

\section{Isolation of mouse bone marrow MSCs}

The isolation of mouse MSCs from the bone marrow of $\mathrm{Balb} / \mathrm{c}$ mice and characterization of MSCs were identified with our previously reported method [10-13]. Mouse MSCs were then cultured in low-glucose DMEM (Sigma-Aldrich) supplemented with 10\% FBS (Thermo Fisher Scientific, Waltham, MA, USA) and 1\% penicillinstreptomycin-glutamine (Thermo Fisher Scientific). The quality and purity of obtained MSCs were assessed, and the isolated MSCs were able to be differentiated into the bone, adipose tissue, and hepatocyte in vitro (see Additional file 1) [10-13]. MSCs were used at passages 8-13 in this study. The study was approved by the Medical and Ethics Committee of our hospital (approval no. 201701193B0).

\section{BMC preparation}

Bone marrow aspiration, concentration, harvesting, and processing were performed as described previously, with modifications $[4,14]$. Before aspiration, a $10-\mathrm{mL}$ syringe was flushed with heparin $(1000 \mu / \mathrm{mL})$. After adequate anesthesia and sterile conditions, the iliac crests of 
rabbits were penetrated with an 18-gauge needle and aspirated. The aspirate was poured into a $10-\mathrm{mL}$ centrifuge tube and spun at $1200 \times g$ for $10 \mathrm{~min}$. After completion of this process, the buffy coat layer and platelet-poor plasma layer were extracted from the centrifuge tube and discarded. Secondary centrifugation was performed at $2400 \times g$ for $6 \mathrm{~min}$. Clear supernatants were removed by aspiration until only $1 \mathrm{~mL}$ was left.

Preparation of MSCs, BMC CM, and BMC-induced MSC CM When mouse bone marrow MSCs reached approximately $80 \%$ confluence in $10-\mathrm{cm}$ dishes, culture medium was changed to $10 \mathrm{~mL}$ DMEM. For the preparation of BMC-induced MSC CM, $200 \mu \mathrm{L}$ BMC was added to cultures of MSCs. For the preparation of BMC CM, $200 \mu \mathrm{L}$ BMC was added to $10-\mathrm{cm}$ dishes containing $10 \mathrm{~mL}$ DMEM. All culture media were separated after $48 \mathrm{~h}$ and used as MSC CM, BMC-induced MSC CM, and BMC CM. This procedure was repeated three times; CM from three different time points was pooled and filtered through a $0.22-\mu \mathrm{m}$ filter (Millex-GP syringe filter; Millipore, Billerica, MA, USA). Pure DMEM was used as a control medium.

\section{Rabbit ear HS model}

The rabbit ear HS model was established as described previously [15]. Briefly, 12 adult female New Zealand albino rabbits (each weighing $3-4 \mathrm{~kg}$ ) were given anesthesia under sterile conditions in preparation for wounding based on a protocol approved by the Animal Care and Experiment Committee of Chang Gung Memorial Hospital. Circular, full-thickness, 1-cm wounds were made to the bare cartilage on the ventral surface of each ear, and the epidermis, dermis, and perichondrium were carefully removed (Fig. 1a). The wounds were covered with neomycin ointment and cleaned of secretions the next day. Two weeks after the operation, the margin of the wound showed obvious protrusions as an indicator of HS formation. Two hundred microliters of DMEM, MSC CM, BMC CM, or BMC-induced MSC CM was injected into each lesion on days 14, 21, and 28 after the operation. We excluded samples with infected or necrotic wounds.

\section{Evaluation of scars}

Photographs were acquired every week to observe external changes in wounds after the operation. The reepithelization rate was recorded and analyzed using Image J software (National Institutes of Health, Bethesda, MD, USA).

\section{Histology and immunohistochemistry}

Scars were harvested for histological detection 35 days after the operation, bisected, and immediately fixed in
$10 \%$ formalin. The scars were embedded in paraffin and cut into sections. For analysis of the scar evaluation index (SEI), sections were stained with hematoxylin and eosin, examined under a microscope (Nikon Eclipse E400; Nikon, Tokyo, Japan), and evaluated with a digital image analysis system (Image J). Measurements were performed twice by a blinded examiner, and average values were used. Further evaluation of the collagen fiber arrangement was carried out using Masson trichrome staining. Briefly, the sections were fixed in Bouin's solution for $1 \mathrm{~h}$ at $56^{\circ} \mathrm{C}$ to improve staining quality and then rinsed in running tap water for 5-10 min to remove yellow color. Samples were then stained with Weigert's iron hematoxylin working solution for $10 \mathrm{~min}$, rinsed in running warm tap water for $10 \mathrm{~min}$ and Biebrich scarletacid fuchsin solution for $10-15 \mathrm{~min}$, and placed in $1 \%$ phosphomolybdic-phosphotungstic acid solution for 10$15 \mathrm{~min}$. The sections were then stained in aniline blue solution for $5 \mathrm{~min}$ and rinsed in 1\% acetic acid solution for $2 \mathrm{~min}$. Collagen fibers were stained blue. Keratin and muscle fibers were stained red. The cell cytoplasm and nuclei were stained light pink and dark brown, respectively. Regions were randomly selected and photographed.

\section{Treatment of scar-derived fibroblasts with MSCs, BMC, and BMC-induced MSC CM}

Adherent scar-derived fibroblasts were incubated in serum-free medium containing transforming growth factor TGF $\beta 1$ (10 ng/mL; Sigma-Aldrich; cat. no. H8541) for $24 \mathrm{~h}$ then switched the culture medium to DMEM, MSC CM, BMC CM, or BMC-induced MSC CM for 72 h. Cells were then subjected to comparative analyses.

\section{Cellular proliferation}

A Cell Counting Kit-8 (CCK-8; Sigma-Aldrich) was used to determine the extent of fibroblast proliferation. Fibroblasts were seeded in 96-well plates at an initial concentration of 5000 cells/well. After stimulated with TGF- $\beta 1$ (10 $\mathrm{ng} / \mathrm{mL})$ for $24 \mathrm{~h}$, then shift the culture medium to DMEM, MSC CM, BMC CM, or BMC-induced MSC CM. CCK- 8 reagent was added for $2 \mathrm{~h}$ after incubation at $37^{\circ} \mathrm{C}$ for $0,24,48$, or $72 \mathrm{~h}$. The absorption rate and reference wavelength were measured at $450 \mathrm{~nm}$.

\section{Collagen gel contraction assay}

After stimulated with TGF- $\beta 1$ for $24 \mathrm{~h}$, scar-derived fibroblasts were seeded in 24-well plates at a density of $2 \times 10^{5}$ cells $/ \mathrm{mL}$ in $0.6 \mathrm{~mL}$ DMEM, MSC CM, BMC CM, or BMC-induced MSC CM supplemented with antibiotics, and $1 \mathrm{mg} / \mathrm{mL}$ acid-extracted collagen I from rat tails (Sigma-Aldrich) was added to different wells. The cells were cultured at $37^{\circ} \mathrm{C}$ for $20 \mathrm{~min}$ to allow collagen polymerization. Then, $0.6 \mathrm{~mL}$ of the different treatment media was added to each group. After incubation at 


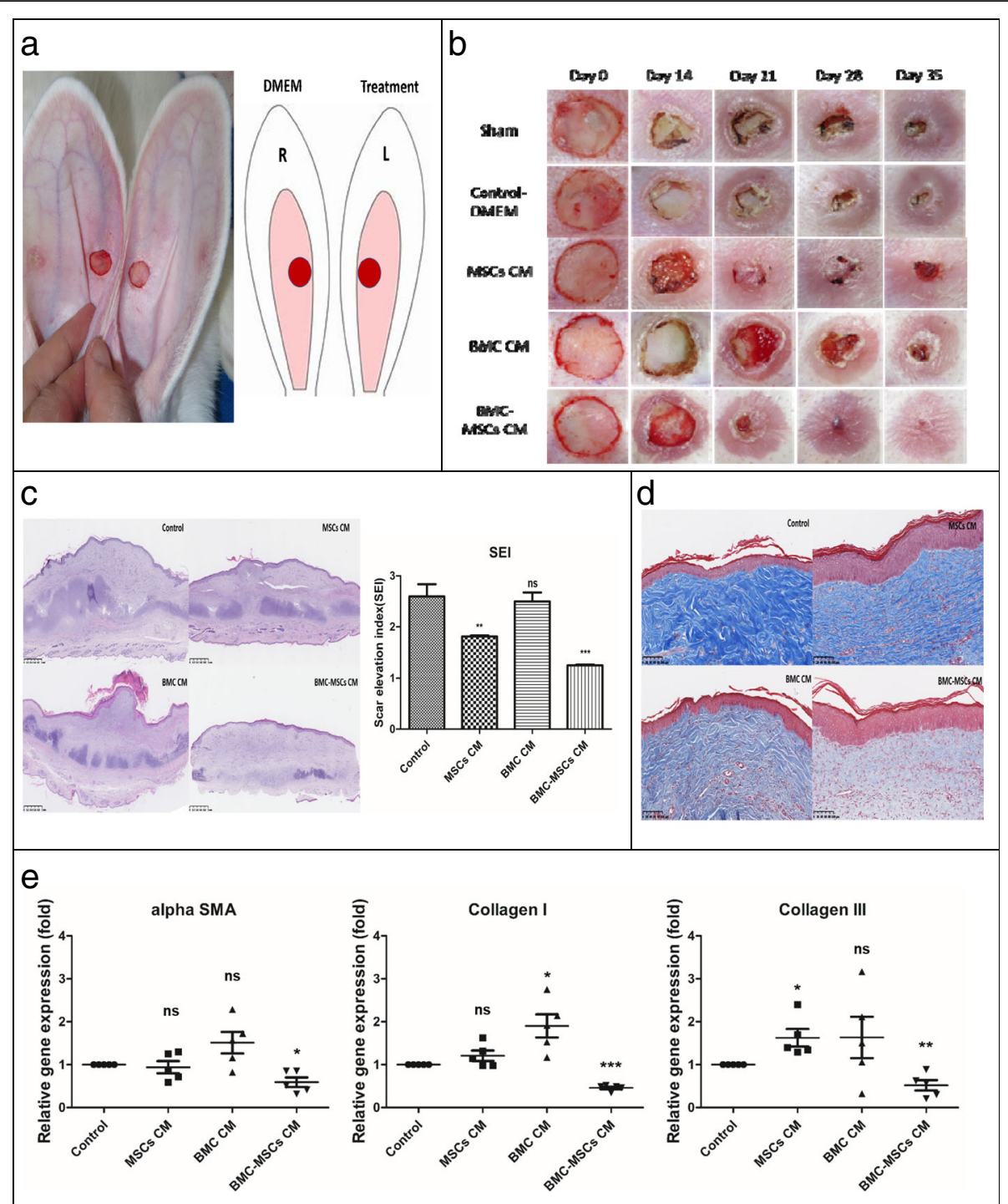

Fig. 1 The rabbit ear HS model. BMC-induced MSC CM accelerated wound healing and prevented HS formation in our rabbit ear model. a Experimental design. Four groups of rabbits were used. MSC CM, BMC CM, or BMC-MSC CM was administered to the left ears. In one set of rabbits, the left ears were left untreated as a sham group. The right ears of all groups received a DMEM injection as an internal control. $\mathbf{b}$ Images of gross examination. c Histological analysis of wounds and scar elevation index (SEI) measurement. Cells were stained with hematoxylin and eosin. Original magnification $\times 40$. The SEl was measured using cross-sections of the central area of the wound. $\mathbf{d}$ Masson trichrome staining was used for the evaluation of collagen fiber organization. e Effects of MSC CM, BMC CM, and BMC-induced MSC CM on a-SMA, collagen type I, and collagen III mRNA expression, as measured by quantitative polymerase chain reaction using GADPH as a housekeeping gene and internal standard control. The data are shown as means \pm standard errors of the means. ${ }^{*} P<0.05 ;{ }^{* *} P<0.01 ;{ }^{* * *} P<0.001$. MSC CM, mesenchymal stem cell-conditioned medium; BMC CM, bone marrow concentrate; DMEM, Dulbecco's modified Eagle's medium; L, left; R, right

$37^{\circ} \mathrm{C}$ for $60 \mathrm{~min}$, the gels were released from the plates by tilting the plates. Contraction of collagen gels was monitored by measuring the gel area at $3,6,9,12$, and $24 \mathrm{~h}$. Data are presented means \pm standard errors of the means (SEMs) of three independent experiments, conducted in triplicate.

\section{Immunofluorescence analysis}

Scar-derived fibroblasts were seeded at a density of 10,000 cells $/ \mathrm{cm}^{2}$. The cells were then incubated in serum-free medium containing transforming growth factor TGF $\beta 1(10 \mathrm{ng} / \mathrm{mL})$ for $24 \mathrm{~h}$ then shift the culture medium to DMEM, MSC CM, BMC CM, or BMC-induced MSC CM. For $\alpha$ smooth muscle actin $(\alpha-S M A)$ staining, cells were incubated with primary antibodies against $\alpha$-SMA $(1 \mu \mathrm{g} / \mathrm{mL}$; Invitrogen $)$ for $75 \mathrm{~min}$ at $25^{\circ} \mathrm{C}$, followed by incubation with a fluorescein isothiocyanate-488-conjugated secondary antibody. Cells were then stained with 4,6-diamidino-2phenylindole (DAPI; $1 \mu \mathrm{g} / \mathrm{mL} ; \quad$ Sigma-Aldrich) for 
nuclear staining. Immunofluorescence signals were captured using a confocal microscope (LSM 510; METALaser Scanning Microscope; Zeiss).

\section{Western blotting}

Immunoblot procedures were performed as described previously [16]. As primary antibodies, mouse anti- $\alpha-$ SMA $(2 \mu \mathrm{g} / \mathrm{mL}$; Invitrogen), anti-GADPH $(0.05 \mu \mathrm{g} / \mathrm{mL}$; Sigma-Aldrich), rabbit anti-collagen I (1:1000; Abcam, Cambridge, UK; cat. no. ab34710), rabbit anti-collagen III (1:5000; Abcam; cat. no. ab7778), rabbit antifibronectin (1:1000; Abcam; cat. no. 1b45688), antimatrix metalloproteinase (MMP)-1 (1:1000; Abcam; cat. no. ab134184), and anti-MMP-13 (1:1000; Abcam; cat. no. ab51072) were used. Anti-mouse IgG (1:50000; Sigma-Aldrich) and anti-rabbit IgG (1:10000 SigmaAldrich) were used as secondary antibodies. Photographs were acquired with an Amersham Imager 600 (GE Healthcare). Densitometric analysis was performed using Image Quant TL software. Expression of proteins of interest was normalized to the expression of GADPH.

RNA isolation, quantitative real-time reverse transcription polymerase chain reaction (qRT-PCR), and preparation for RNA-seq

qRT-PCR was performed as previously reported [16]. Briefly, scar-derived fibroblasts were collected after $72 \mathrm{~h}$ of incubation with different treatments, and rabbit scars were harvested on day 35 post-operation. Total RNA was extracted using an RNA isolation kit (Qiagen, Hilden, Germany), and RNA purity was evaluated by calculating the A260/A280 ratio by nanodrop spectrophotometer, aiming for a value of 1.8-2.1. The primer pairs used for gene amplification from the cDNA template are listed in Additional file 2. The results from three independent reactions were used to determine the relative expression levels of the target genes, which were normalized to the expression level of glyceraldehyde 3-phosphate dehydrogenase (GAPDH) as a control. As for RNA sequencing, sample quality control of RNA integrity was performed using an Agilent 2100 BioanalyzerRNA 6000 Nano kit. High-quality RNA was selected based on samples with an RNA integrity number over 9.5. Qualified samples were then used to construct RNA-seq cDNA libraries following the standard Illumina protocol. cDNA libraries were quantified by qRT-PCR using a Roche LightCycler 480 system and Qubit Fluorometer (Invitrogen). The cDNA libraries were pooled and sequenced on an Illumina NextSeq 500 platform with single-end 76-bp reads.

\section{RNA-seq analysis}

Quality control of RNA-seq reads was performed using FASTX-Toolkit (v0.0.13.2) with high base calling quality (Phred quality score over 20) and RNA read lengths over 35 bases. Reads were mapped to the UCSC human genome hg38 using Tophat2 (v2.1.0) [17]. The transcripts and abundances were assembled and estimated by Cufflinks (v2.2.1) [18]. Mapping reads of each gene were counted by HTSeq (v0.11.1) [19]. Differential gene expression was analyzed using DESeq2 (v1.22.2) [20]. Genes with low baseMean values $(\leq 10)$ were filtered. Differences with $P$ values of less than 0.05 were considered statistically significant. Genes were identified as upregulated or downregulated if the fold change between case and control sample was greater than $|1.3|$.

\section{Gene ontology (GO) and enrichment analysis}

To study the functions of differentially expressed genes (DEGs), functional groups and pathways encompassing the DEGs were identified based on GO and Kyoto Encyclopedia of Genes and Genomes Pathway analysis using the Database for Annotation, Visualization, and Integrated Discovery (v.6.8) software [21, 22]. The threshold was set as modified Fisher exact $P$ value (EASE score) $\leq 0.1$, and the top 20 functional terms sorted by modified Fisher exact $P$ values are shown.

\section{Statistical analysis}

Statistical differences were calculated using two-tailed Student's $t$ tests compared to the control group. Differences with $P$ values of less than 0.05 were considered statistically significant. For RNA sequencing and GO analysis, the statistical analysis was described as above.

\section{Results}

BMC-induced MSC CM significantly improved wound healing and reduced HS formation after wound healing in a rabbit ear model

The experimental groups and study design are shown in Fig. 1a. Rabbit ears were treated with DMEM, MSC CM, BMC CM, or BMC-induced MSC CM on days 14, 21, and 28 after the operation. Rabbit ears treated with BMC-induced MSC CM healed significantly faster than rabbit ears in the other groups, and complete closure occurred on day 28 after the operation. However, sham-, DMEM-, MSC CM-, and BMC CM-treated rabbit ears did not show complete re-epithelization within the time course tested (Fig. 1b). An additional file shows the time of rabbit ear wound healing in more detail (see Additional file 3). On postoperative day 35, hematoxylin-and-eosin staining of cross-sections of HSs showed that BMC-induced MSC CM- and MSC CM-treated scars were flatter and thinner. However, DMEM- and BMC CM-treated scars were obviously thickened, with slight contraction (Fig. 1c). The SEIs in MSC CM- and BMC-induced MSC CM-treated scars were much lower than those in internal controls. However, there were no significant differences between DMEM- and BMC CM-injected scars (Fig. 1c). 
BMC-induced MSC CM attenuated scar-forming profibrotic activity and fibroblast activation in a rabbit ear model

Profibrotic events and fibroblast activation are key processes in HS formation and can be measured by evaluating collagen deposition and myofibroblast numbers. Masson trichrome staining revealed that collagen fibers were dense and irregularly arranged in DMEM- and BMC CMtreated scars. Conversely, collagen deposition was reduced and well arranged in MSC CM- and BMC-induced MSC CM-treated scars (Fig. 1d). Detection of myofibroblasts using $\alpha$-SMA showed that $\alpha-S M A$ gene expression in scar tissue was reduced in BMC-induced MSC CM-treated scars compared with those in DMEM- and MSC CMtreated scars on day 35 post-operation (Fig. 1e). Collagen I and collagen III gene expression levels were also significantly reduced in BMC-induced MSC CM-treated scars compared with that in DMEM-treated scars (Fig. 1e).

\section{BMC-induced MSC CM promoted the balance of ECM turnover in human HS-derived fibroblasts}

During normal wound healing, matrix metalloproteinase (MMP) expression modulates matrix turnover [23]. MMPs catalyze the hydrolysis of ECM molecules [24], and MMP-13 and MMP-1 expression levels vary between normal and abnormal scar tissues [25]. Therefore, we examined the effects of BMC-induced MSC CM on ECM synthesis in active human HS-derived fibroblasts. BMC-induced MSC CM decreased collagen I, collagen III, and fibronectin expression at both the transcriptional and translational levels (Fig. 2). Moreover, BMC-induced MSC CM significantly increased MMP-1 expression and decreased MMP-13 expression.

To further determine the functional consequences of these effects, we analyzed GO data for BMC-induced MSC CMtreated HSs from three human samples. Genes regulating ECM formation, ECM organization, extracellular structure organization, collagen fibril organization, and collagen fibril binding, such as collagen type VIII alpha 2 (COL8A2), collagen type XI alpha 1 (COL11A1), collagen Type XIV Alpha 1 (COL14A1), collagen Type XVI alpha 1 (COL16A1), extracellular matrix protein 2 (ECM2), matrilin 3 (MATN3), and matrix metalloproteinase 13 (MMP-13), as well as related pathways were downregulated (Fig. 5d, f). Additionally, $M M P-1$ was upregulated (Fig. 5e). An additional file shows RNA sequence data in more detail (see Additional file 4). Taken together, our results showed that BMC-induced MSC $\mathrm{CM}$ reversed the abnormal expression profiles of collagens and MMPs in human HS-derived fibroblasts.

\section{BMC-induced MSC CM reduced the contractile ability of $\mathrm{HS}$ in collagen gels}

To investigate the effects of BMC-induced MSC CM on the contractile ability and functions of HSs, we next examined the properties of human HS-derived fibroblasts in three-dimensional (3D) fibroblast function assays. After edges were liberated from array wells, we observed collagen lattice contraction at 3, 6, 9, 12, and 24h. BMC-induced MSC CM had significant timedependent inhibitory effects on the contractile ability of HSs (Fig. 3a, b). No differences in collagen contraction were observed at 3 and $6 \mathrm{~h}$ between any of the groups. At 9, 12, and 24h, BMC-induced MSC CM induced the lowest collagen contraction, followed by BMC CM, MSC CM, and DMEM. BMC-induced MSC CM and BMC $\mathrm{CM}$ resulted in significantly lower collagen matrix contraction compared with that in the control group at 12 and $24 \mathrm{~h}$. Thus, MSC CM, BMC CM, and BMC-induced MSC CM efficiently inhibited the contractile ability of HSs in $3 \mathrm{D}$ collagen gels, and BMC-induced MSC CM was more effective than the other treatments.

\section{BMC-induced MSC CM reversed the transformation of fibroblasts to myofibroblasts in human HS-derived fibroblasts treated with TGF- $\beta 1$}

In wound healing, TGF- $\beta 1$ induces the differentiation of fibroblasts into myofibroblasts, which contract the wound and facilitate remodeling of the ECM [24, 26, 27]. To investigate the phenotype of fibroblasts isolated from human HSs, we examined $\alpha$-SMA expression in these cells by flow cytometry. Only $60-80 \%$ of fibroblasts from HSs were positive for $\alpha$-SMA. After stimulation with TGF- $\beta 1(10 \mathrm{ng} / \mathrm{mL})$ for $24 \mathrm{~h}$, more than $98 \%$ of fibroblasts from HSs were positive for $\alpha$-SMA (Fig. 4a). $\alpha-S M A$ gene expression was also significantly increased compared with that in untreated cells (Fig. 4b). To further investigate the effects of BMC-induced MSC CM on fibroblast activation in vitro, we evaluated the levels of $\alpha$-SMA expression in cultured HSs treated with 10 $\mathrm{ng} / \mathrm{mL}$ TGF- $\beta 1$ for $24 \mathrm{~h}$ followed by different treatments for $72 \mathrm{~h}$. We found that $\alpha$-SMA gene and protein expression were noticeably reduced in the BMC-induced MSC CM-, BMC CM-, and MSC CM-treated groups compared with that in the DMEM-treated group (Fig. 2). Immunostaining and flow cytometry analyses also showed that BMC-induced MSC CM suppressed TGF$\beta 1$-induced $\alpha$-SMA expression and decreased $\alpha$-SMApositive fibroblasts by $10.4 \% \pm 2.2 \%(P<0.01)$ in cultured HSs (Fig. 4c, d). These findings suggested that BMCinduced MSC CM could reverse the activation of fibroblasts induced by the inflammatory factor TGF- $\beta 1$.

\section{BMC-induced MSC CM accelerated cell proliferation in} human HS-derived fibroblasts and our rabbit ear model CCK-8 assays showed that the proliferation of human HS-derived fibroblasts increased after incubation with BMC CM or BMC-induced MSC CM for 24,48 , or $72 \mathrm{~h}$, compared with that in cells incubated with DMEM or 


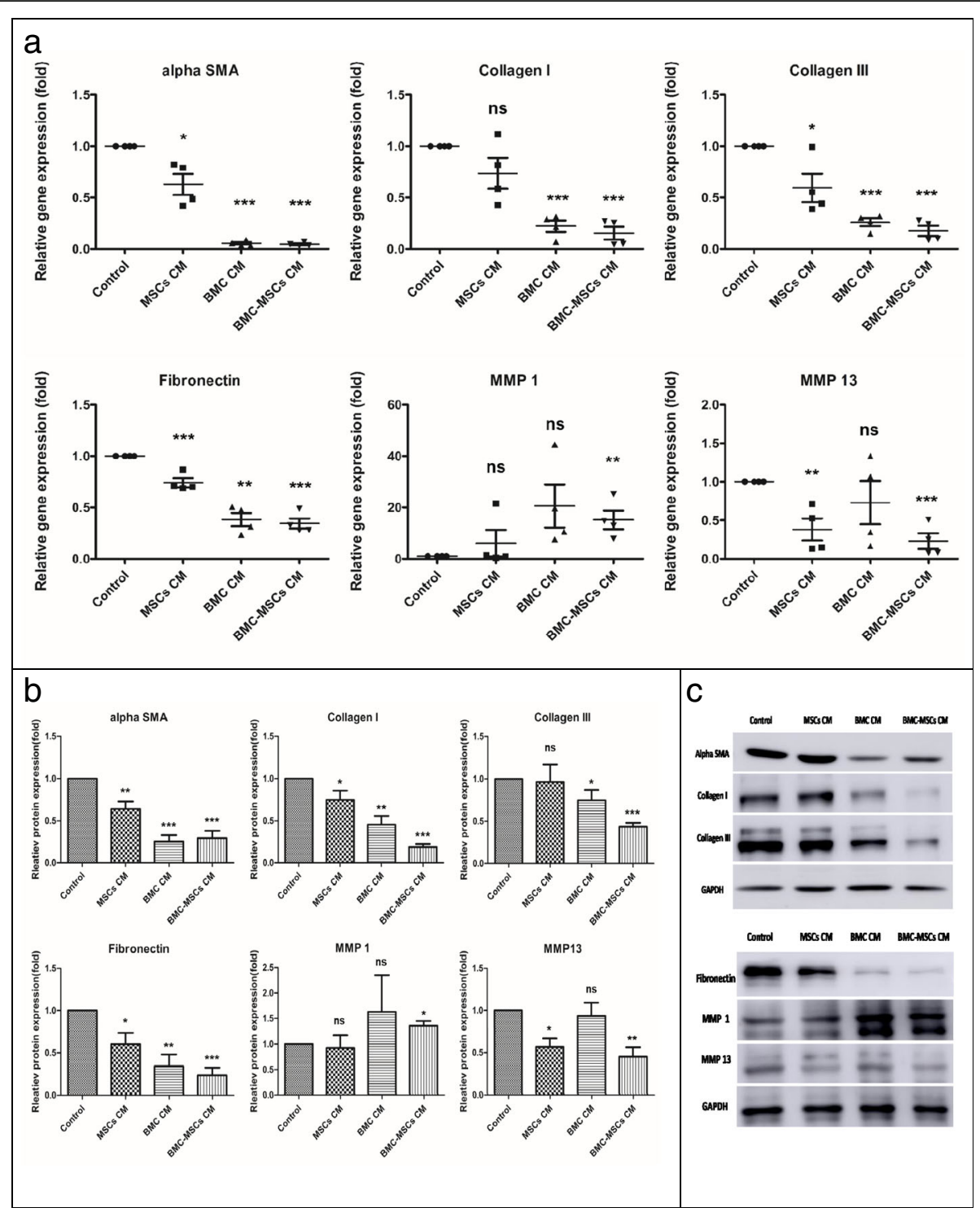

Fig. 2 Effects of MSC CM, BMC CM, and BMC-induced MSC CM on cultured primary HSs. a qRT-PCR analysis of a-SMA, collagen I, collagen III, fibronectin, MMP-1, and MMP-13 in HSs treated for $72 \mathrm{~h}$ with different stimuli. Expression is reported relative to that of GAPDH. Each data point was normalized to GAPDH expression and then the value of the control (DMEM), which was arbitrarily set as 1. b, $\mathbf{c}$ Western blot analysis and quantitative data for a-SMA, collagen I, collagen II, fibronectin, MMP-1, and MMP-13 after treatment with DMEM, MSC CM, BMC CM, and BMCinduced MSC CM for $72 \mathrm{~h}$. GAPDH served as a control for equal protein loading. Histogram summarizing the results in $\mathbf{b}$. Results were from three independent experiments using cells from four patients with HSs. The data are shown as means \pm standard errors of the means. ${ }^{*} P<0.05$; ${ }^{* *} P<0.01 ;{ }^{* *} P<0.001$

MSC CM (Fig. 5a). Additionally, flow cytometry showed that in the BMC $\mathrm{CM}$ and $\mathrm{BMC}$-induced MSC CM groups, the number of cells in the $G_{0} / G_{1}$ phase was significantly decreased, whereas the numbers of cells in the $\mathrm{S}$ and $\mathrm{G}_{2} / \mathrm{M}$ phases were significantly increased (Fig. $5 \mathrm{~b}$ ). Furthermore, GO analysis revealed genes related to cell proliferation and DNA replication, such as SCLinterrupting locus protein (STIL), cell division cycle (CDC), kinesin family member (KIF), marker of proliferation Ki-67, and DNA replication complex GINS protein, were upregulated in BMC-induced MSC CMtreated HSs. Pathways related to cell division and mitosis were upregulated in this cohort as well (Fig. 5c, e). Interestingly, in rabbit ear HSs, the BMC-induced MSC CMtreated group showed high levels of DNA oxidation compared with those in the DMEM-, MSC CM-, and BMC CM-treated groups, as measured by 8-oxodG assays (Additional file 5), suggesting that the resident cells in rabbit ear wounds underwent DNA replication and proliferation. 

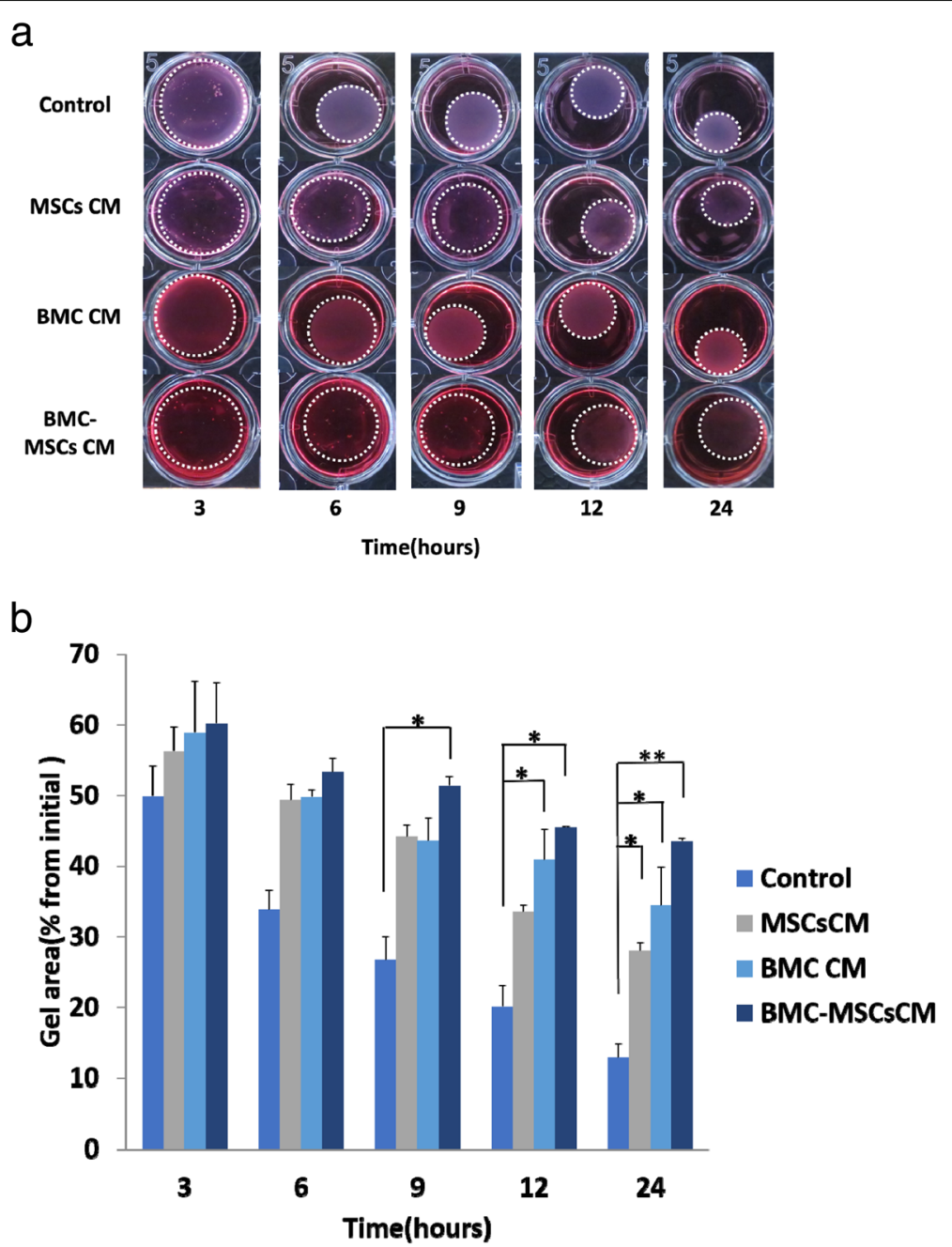

Fig. 3 Effects of MSC CM, BMC CM, and BMC-induced MSC CM attenuated the contractile ability of human hypertrophic scar-derived fibroblasts in $3 \mathrm{D}$ collagen gels. $\mathbf{a}$, $\mathbf{b}$ Representative images and quantification of collagen gel contraction after treatment with different samples for 3, 6, 9, 12 , or $24 \mathrm{~h}$. The white spots outline the gels $(n=3)$. Values are shown as means \pm standard errors of the means. ${ }^{*} P<0.05,{ }^{*} P<0.01$

\section{Discussion}

Several factors contribute to HS formation during the wound healing process; for example, prolonged wound healing, trauma mechanism and severity, and mechanical tension on the wound are leading causes of HS formation [28]. Importantly, the timing of wound re-epithelization can decide the outcome of the healing process [29]. Slow epithelization of tissues over the wounded area results in HS formation, whereas rapid epithelization results in regeneration $[30,31]$. Delayed wound healing increases the chance of HS formation. Our results showed that BMCinduced MSC CM accelerated re-epithelization and resulted in much lower SEI, more regular collagen arrangement, and decreased $\alpha$-SMA, collagen type I, and collagen type III expression in our rabbit ear model. Moreover, cell proliferation was elevated in the BMC-induced MSC CM group.
The wound healing process consists of three phases: inflammation, proliferation, and remodeling. During the initial wound inflammation stage, neutrophils secrete reactive oxygen species (ROS) to sterilize the wound. In tissues with prolonged inflammation, ROS are overproduced, and T cells and macrophages are dysfunctional $[1,32]$. During the proliferation stage, TGF- $\beta 1$ plays a critical role. TGF- $\beta 1$ is a collagen-stimulating factor that can inhibit MMP expression and contribute to ECM deposition within wounds [33, 34]. TGF- $\beta 1$ can also stimulate the transformation of fibroblasts into myofibroblasts at a later stage, which can narrow the margin of the wound by contraction and therefore accelerate re-epithelization. However, a prolonged proliferation stage will lead to excessive irregularly arranged collagen bundles in the ECM and cause tensed, over contracted scars as additional pathological characteristics of HSs [35, 36]. During the remodeling stage, type III collagen, which is prevalent during proliferation, is replaced by type I collagen. 


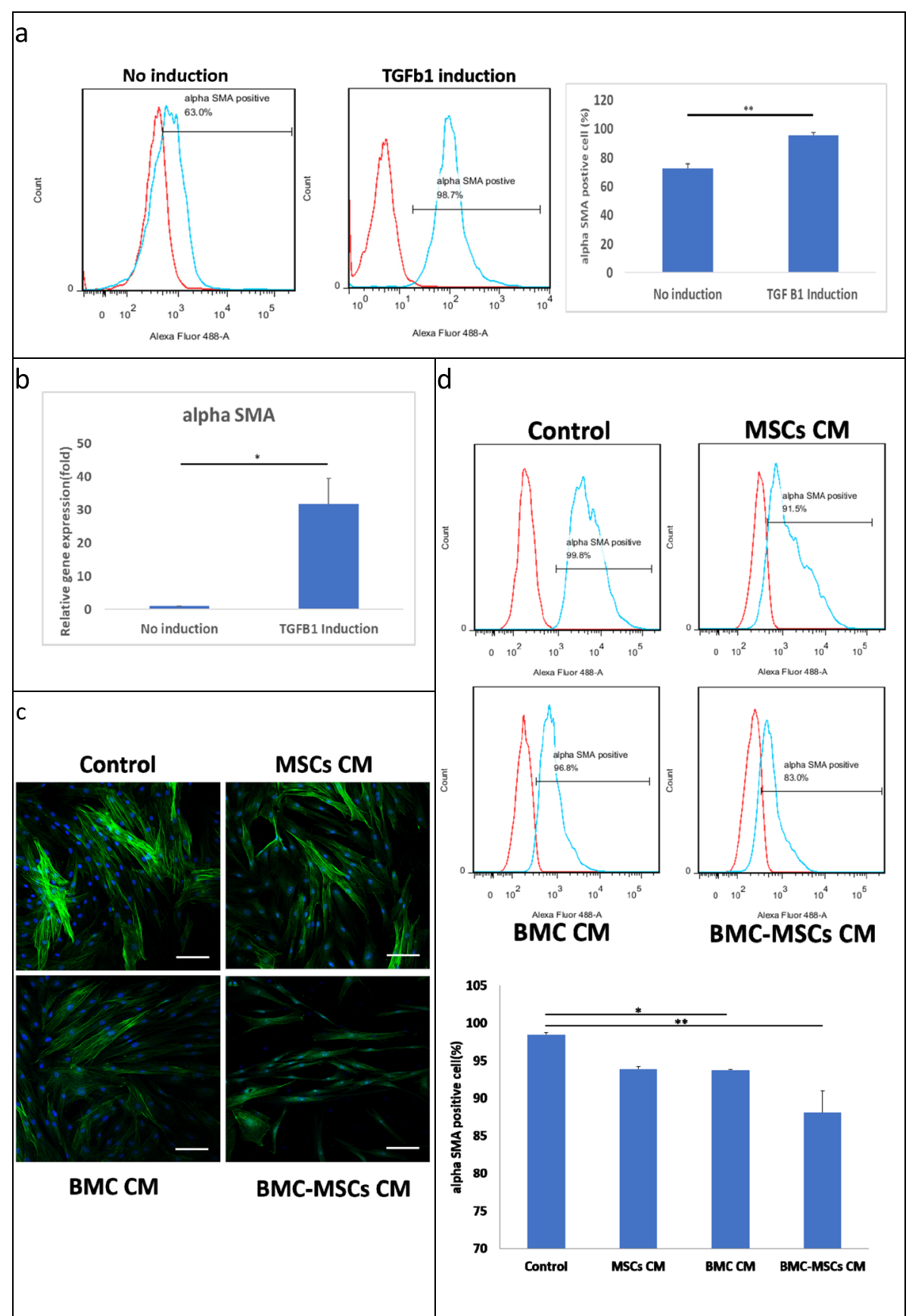

Fig. 4 BMC-induced MSC CM attenuated the activation of TGF- $\beta 1$-stimulated myofibroblasts in human hypertrophic scar-derived fibroblasts. a Flow cytometry analysis and quantitative data for a-SMA-positive cells after TGF- $\beta 1$ stimulation for $24 \mathrm{~h}$. $\mathbf{b}$ Expression of the $\alpha$-SMA gene after TGF- $\beta 1$ stimulation for $24 \mathrm{~h}$. c Immunofluorescence staining for a-SMA expression in HSs stimulated with TGF- $\beta 1$ for $24 \mathrm{~h}$ and treated with DMEM, MSC CM, BMC CM, and BMC-induced MSC CM for $72 \mathrm{~h}$. Scale bars = $100 \mu \mathrm{m}$. DAPI, 4,6-diamidino-2-phenylindole; MSC CM, mesenchymal stem cell-conditioned medium; BMC CM, bone marrow concentrate; DMEM, Dulbecco's modified Eagle's medium; SMA, smooth muscle actin. $\mathbf{d}$ Flow cytometry analysis and quantitative data for a-SMA-positive cells after TGF- $\beta 1$ stimulation for $24 \mathrm{~h}$, followed by treatment with DMEM, MSC CM, BMC CM, and BMC-induced MSC CM for $72 \mathrm{~h}(n=4)$. Values are denoted as means \pm standard errors of the means. ${ }^{*} P<0.05$, ${ }^{* *} P<0.01$

Additionally, disorganized collagen fibers are rearranged, crosslinked, and aligned along tension lines. All protein components and the ECM are subjected to degradation and modification. The most important enzymes in ECM remodeling are MMPs [37]. If protein components in the ECM fail to be degraded, HSs will develop. Our results showed that BMC-induced MSC CM could decrease $\alpha$ SMA gene and protein expression and reverse 


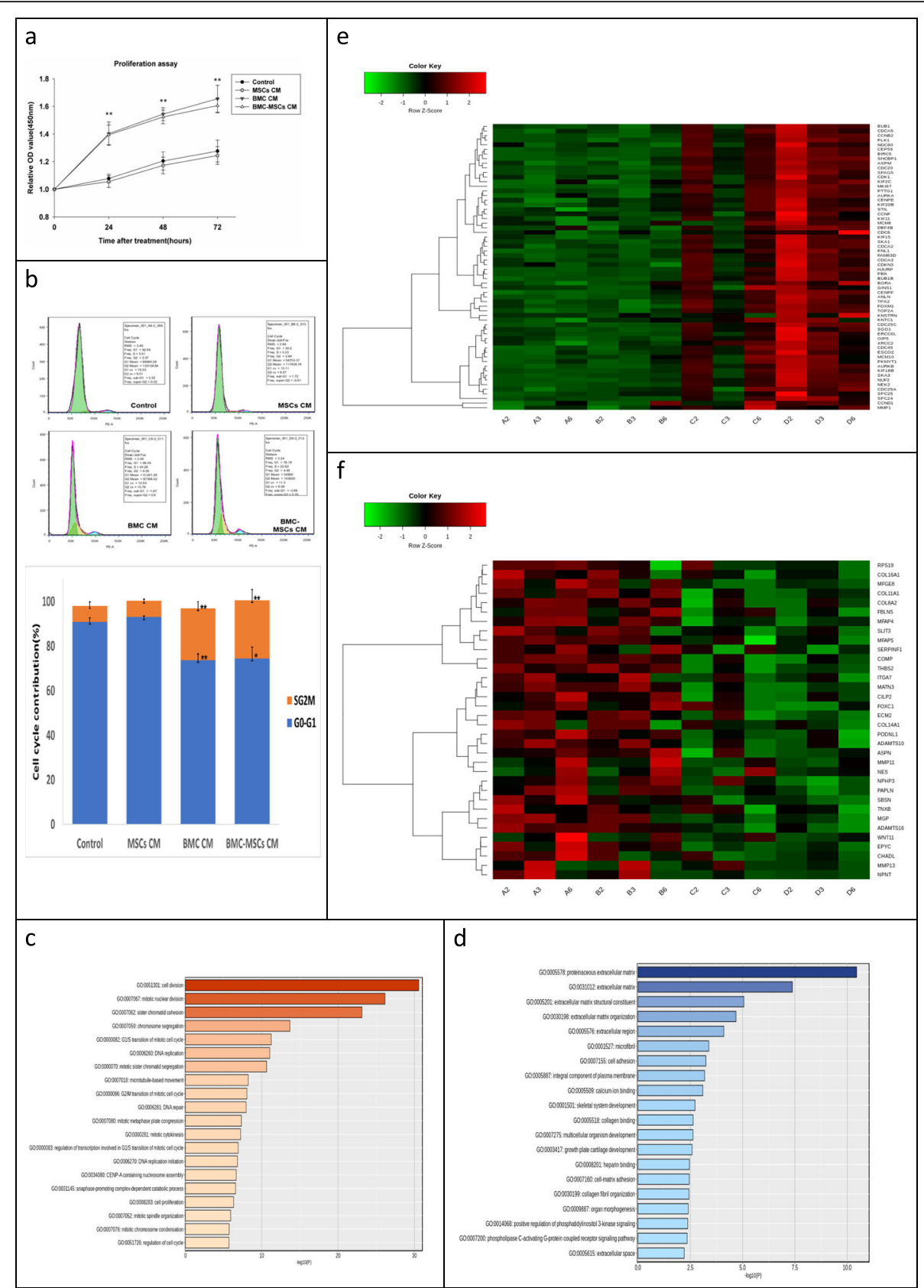

Fig. 5 BMC-induced MSC CM accelerated cell proliferation in cell culture and in our rabbit ear model. a HS-derived fibroblasts were cultured in MSC CM, BMC CM, and BMC-induced MSC CM for $72 \mathrm{~h}$. Cell viability was determined by CCK-8 assays every $24 \mathrm{~h}$ and compared with DMEMtreated samples. b Determination of cell cycle distribution by flow cytometry in HS-derived fibroblasts after culture with DMEM, MSC CM, BMC CM, or BMC-induced MSC CM for 72 h. c-f RNA-seq analysis of HSs treated with DMEM or BMC-induced MSC CM for $72 \mathrm{~h}$. Genes that were differentially expressed between DMEM and BMC-induced MSC CM are shown as up- or downregulated. $\mathbf{c}$, $\mathbf{d}$ GO enrichment for up- and downregulated pathways. e, f Heatmap of hierarchical clustering indicated differentially expressed genes (rows) from three patients (fold change $>1.3, P<0.05$ ). Red indicates upregulation, and green indicates downregulation. Data are means \pm standard errors of the means. ${ }^{*} P<0.05$ compared with DMEM. A, DMEM; B, MSC CM; C, BMC CM; D, BMC-MSC CM; 2, 3, 6, no. of patient

myofibroblast activation stimulated by TGF- $\beta 1$. BMCinduced MSC CM also maintained tissue homeostasis by regulating the turnover of the ECM. Gene and protein expression of MMP-1 was elevated, whereas that of MMP-13 was decreased in HSs after treatment with BMC-induced
MSC CM. The expression of collagen types I and III was also decreased after treatment with BMC-induced MSC $\mathrm{CM}$ in cultured HS-derived fibroblasts and our rabbit ear model. Overall, our observations suggested that BMCinduced MSC CM may suppress HS formation by 
modulating the proliferation and remodeling stages, reducing profibrotic gene activation, reversing myofibroblast activation, and facilitating ECM turnover and degradation; these changes together attenuated HS formation.

MSCs have anti-scarring effects by promoting wound healing. Additionally, MSCs have strong therapeutic effects on HSs because these cells can interact with many types of cells in the microenvironment through paracrine signaling pathways [38]. Components secreted from MSCs, including molecules and extracellular vesicles, are responsible for these therapeutic effects and have both local and distant effects. However, this process is not spontaneous and can be altered by different stimuli [39]. Several studies have shown that MSC-based strategies should be combined with appropriate differentiation and growth factors to obtain the maximum therapeutic effects from MSCs [39-42]. Different preconditioning strategies can enhance the secretion of therapeutic factors into the MSC culture medium $[42,43]$. Thus, it is necessary to determine which culture conditions allow MSCs to produce differing sets of trophic factors [39].

$\mathrm{BMC}$ is currently often used for regenerative medicine because it contains a heterogenous mixture of cells, including small amount MSCs, hematopoietic cells, and platelets, as well as bioactive molecules, such as plateletderived growth factor, basic fibroblast growth factor, insulin-like growth factor-I, and vascular endothelial growth factor [3, 41, 44]. BMC acts as a storage vehicle for a variety of growth factors, thereby enhancing the differentiation of MSCs and stimulating the production of secreted factors from MSCs. We used BMC from rabbits to stimulate mouse MSCs, and the CM we obtained was shown to have significant effects on rabbit ear and human HS fibroblasts, despite the use of mixed species. MSCs are known to have immunomodulatory effects and cross-species immunotolerance [45]; however, the mechanisms mediating the differences between secreted factors from mouse MSCs with and without rabbit BMC stimulation are still unclear. Accordingly, in our future studies, we will investigate the differential contents of CM (BMC-induced MSC CM versus MSCs and/or BMC $\mathrm{CM}$ ) and the contributions of rabbit BMC cells, mouse MSCs, and their derivatives in BMC-induced MSC CM.

\section{Conclusions}

BMC-induced MSC CM accelerated wound healing and attenuated $\mathrm{HS}$ formation in a rabbit ear model. Further analyses indicated that BMC-induced MSC CM had anti-HS effects by increasing cell proliferation, modulating the proliferation and remodeling phases of the wound healing process, reducing profibrotic gene expression, balancing ECM turnover, and reversing myofibroblast activation. Therefore, BMC-induced MSC CM may be a promising candidate for preventing HS formation and treating fibrotic diseases.

\section{Additional files}

Additional file 1: Mouse bone marrow MSC isolation. The femurs and tibias of Balb/c mice were dissected, and the ends of the bones were cut. Approximately $2 \mathrm{~mL}$ of bone marrow aspirate was collected into a syringe containing $4000 \mathrm{U}$ heparin to prevent clotting. The bone marrow sample was washed with phosphate-buffered saline (PBS), and the cells were recovered after centrifugation at $900 \times \mathrm{g}$ for $10 \mathrm{~min}$. The cells were resuspended in $10 \mathrm{~mL}$ PBS followed by filtration through a cell strainer with 70- $\mu \mathrm{m}$ nylon mesh. To isolate the mononuclear cells, the filtered bone marrow cells were added to $10 \mathrm{~mL}$ Ficoll-Paque PLUS density gradient separation medium (density: $1.077 \mathrm{~g} / \mathrm{mL}$ ) and centrifuged at $18{ }^{\circ} \mathrm{C}$ for $30 \mathrm{~min}$ at $1100 \times \mathrm{g}$. The mononuclear cells were collected, washed with PBS, and centrifuged for $10 \mathrm{~min}$ at $900 \times \mathrm{g}$. The cells were resuspended, counted, plated at 200000 cells $/ \mathrm{cm}^{2}$, and cultured in Dulbecco's modified Eagle's medium containing 10\% fetal bovine serum. The medium was replaced every 3 days, and the nonadherent cells were discarded. Cells were harvested at 80-90\% confluence and then expanded. After a series of passages, the attached marrow cells became homogeneous. The quality and purity of obtained MSCs were then assessed by detecting the expressions of CD29, CD44, CD73, CD105, CD106 and Sca1, using flow cytometry (FACScan flow cytometer; Becton Dickinson) $[10,12]$. The isolated MSCs were able to be differentiated into bone, adipose tissue, and hepatocyte in vitro $[10,12]$. (DOC $2510 \mathrm{~kb}$ )

Additional file 2: List of oligonucleotides used for quantitative ReverseTranscriptase polymerase Chain Reaction. (DOC 36 kb)

Additional file 3: Time of rabbit ear wound healing. The affected skin area after the $1 \mathrm{~cm}^{2}$ wound creation. Rabbit ears treated with BMCinduced MSCs CM healed significantly faster than rabbit ears in the other groups, and complete closure occurred on day 28 after operation. However, sham-, DMEM-, MSCs CM-, and BMC CM-treated rabbit ears did not show complete re-epithelization within the time course tested. A twotailed independent t-test was performed to evaluate the significant difference between groups. ${ }^{*} p<0.05$. ${ }^{* *} p<0.01$. Error bar indicates standard deviation. (DOC $173 \mathrm{~kb}$ )

Additional file 4: Raw data of RNA sequence. Detail of differential gene expression and fold of change between case and control. A: DMEM, B: MSCs CM, C: BMC CM, D: BMC-MSCs CM. 2,3,6: No. of patient (XLSX 40 kb)

Additional file 5: DNA oxidation level- 8'-oxodG. Measurement of 8'oxodG in rabbit hypertrophic scars on day 35 after treatment with DMEM, MSCs CM, BMC CM, or BMC-induced MSCs CM. BMC-induced MSCs CM-treated group showed high levels of DNA oxidation compared with those in the DMEM-, MSC CM-, and BMC CM-treated groups. ( $\left.{ }^{*} \mathrm{p}<0.05\right)$ Error bar indicates standard deviation. (DOC $120 \mathrm{~kb}$ )

\section{Abbreviations}

a-SMA: a smooth muscle actin; BMC: Bone marrow concentrate; CCK-8: Cell counting kit-8; CM: Conditioned medium; HS: Hypertrophic scar;

MSC: Mesenchymal stem cell; TGF $\beta 1$ : Transforming growth factor $\beta 1$

\section{Acknowledgements}

The authors acknowledge financial support from the Ministry of Science and Technology, Taiwan (MOST; grant no. MOST 107-2314-B-182A-006 and MOST 108-2321-B-010-006). We appreciate Chien-Wei Lee, PhD's assistance for characterization of BM-MSC.

\section{Authors' contributions}

$\mathrm{CHH}$ was involved in the study design, manuscript writing, and statistical analysis; YWT was involved in performing the experiments (cell studies, qPCR, and western blotting); CYC participated in the immunohistology, immunocytology, and flow cytometry analyses; $\mathrm{KCL}$ and $\mathrm{CHC}$ helped with the processing of the sequencing data, data analyses, interpretation of the results, and additional experiment for the reviewer's suggestion; CST performed RNA sequence sample preparation and helped with the interpretation of the results; $\mathrm{HDH}$ was involved in the interpretation of the RNA sequencing results and helped to design and supervise the RNA sequencing project; CWH performed cellular 8-oxodG analysis using LC-MS/MS and analyzed the 8-oxodG data; KHL performed RNA sequence data visualization; SSC and JYY were surgeons who harvested human hypertrophic scar 
samples and assisted with the animal experiment; OKL designed the study and supervised all of the study. All authors read and approved the final manuscript.

\section{Funding}

Not applicable

\section{Availability of data and materials}

The datasets used and/or analyzed during the current study are available from the corresponding author on reasonable request.

\section{Ethics approval and consent to participate}

Studies involving human participants, human data, or human tissues were approved by the Medical and Ethics Committee of our hospital (IRB no:: 201701193BO). The animals were treated according to protocols approved by Chang Gung Memorial Hospital Animal Care and Use Committee (approval no. 2017070701).

\section{Consent for publication}

Not applicable

\section{Competing interests}

The authors declare that they have no competing interests.

\section{Author details}

'Department of Plastic and Reconstructive Surgery, Chang Gung Memorial Hospital, Chang Gung Medical College and Chang Gung University, Taoyuan, Taiwan. ${ }^{2}$ Institute of Clinical Medicine, National Yang-Ming University, Taipei, Taiwan. ${ }^{3}$ Stem Cell Research Center, National Yang-Ming University, Taipei, Taiwan. ${ }^{4}$ Institute of Molecular Medicine and Bioengineering, National Chiao Tung University, Hsinchu 300, Taiwan. ${ }^{5}$ Department of Biological Science and Technology, Center for Intelligent Drug Systems and Smart Bio-devices (IDS'B), National Chiao Tung University, Hsinchu 300, Taiwan. ${ }^{6}$ Department of Biological Science and Technology, National Chiao Tung University, Hsinchu 300, Taiwan. ${ }^{7}$ Warshel Institute for Computational Biology, School of Life and Health Sciences, School of Sciences and Engineering, The Chinese University of Hong Kong, Shenzhen 518172, China. ${ }^{8}$ Department of Public Health, Chung Shan Medical University, Taichung 402, Taiwan.

\section{Received: 17 May 2019 Revised: 10 August 2019}

\section{Accepted: 13 August 2019 Published online: 28 August 2019}

\section{References}

1. Zhang J, Li Y, Bai X, Li Y, Shi J, Hu D. Recent advances in hypertrophic scar. Histol Histopathol. 2018;33:27-39.

2. Fortier LA, Potter HG, Rickey EJ, et al. Concentrated bone marrow aspirate improves full-thickness cartilage repair compared with microfracture in the equine model. J Bone Joint Surg Am. 2010;92:1927-37.

3. Cassano JM, Kennedy JG, Ross KA, Fraser EJ, Goodale MB, Fortier LA. Bone marrow concentrate and platelet-rich plasma differ in cell distribution and interleukin 1 receptor antagonist protein concentration. Knee Surg. Sports Traumatol. Arthrosc. 2018;26:333-42.

4. Chahla J, Mannava S, Cinque ME, Geeslin AG, Codina D, LaPrade RF. Bone marrow aspirate concentrate harvesting and processing technique. Arthroscopy Techniques. 2017;6:e441-5.

5. Ponemone V, Gupta S, Sethi D, et al. Safety and effectiveness of bone marrow cell concentrate in the treatment of chronic critical limb ischemia utilizing a rapid point-of-care system. Stem Cells Int. 2017;2017:4137626.

6. Centeno C, Sheinkop M, Dodson E, et al. A specific protocol of autologous bone marrow concentrate and platelet products versus exercise therapy for symptomatic knee osteoarthritis: a randomized controlled trial with 2 year follow-up. J Transl Med. 2018;16:355.

7. Zhou Y, Yuan J, Zhou B, et al. The therapeutic efficacy of human adipose tissue-derived mesenchymal stem cells on experimental autoimmune hearing loss in mice. Immunology. 2011;133:133-40.

8. Hong SJ, Traktuev DO, March KL. Therapeutic potential of adipose-derived stem cells in vascular growth and tissue repair. Curr. Opin. Organ Transplant. 2010;15:86-91.

9. Babu M, Diegelmann R, Oliver N. Fibronectin is overproduced by keloid fibroblasts during abnormal wound healing. Mol Cell Biol. 1989;9:1642-50.

10. Wu HH, Lee OK. Exosomes from mesenchymal stem cells induce the conversion of hepatocytes into progenitor oval cells. Stem Cell Res Ther. 2017;8:117.
11. Jiang WC, Cheng YH, Yen MH, Chang Y, Yang WW, Lee OK. Cryo-chemical decellularization of the whole liver for mesenchymal stem cells-based functional hepatic tissue engineering. Biomaterials. 2014;35:3607-17.

12. Lee $\mathrm{CW}$, Huang WC, Huang HD, et al. DNA methyltransferases modulate hepatogenic lineage plasticity of mesenchymal stromal cells. Stem Cell Reports. 2017;9:247-63.

13. Yen MH, WU YY, Liu YS, Rimando M, Ho JH, Lee OK. Efficient generation of hepatic cells from mesenchymal stromal cells by an innovative biomicrofluidic cell culture device. Stem Cell Res Ther. 2016;7:120.

14. Nishimoto S, Kawai K, Tsumano T, Fukuda K, Fujiwara T, Kakibuchi M. Impacts of bone marrow aspirate and peripheral blood derived platelet-rich plasma on the wound healing in chronic ischaemic limb. J Plastic Surg Hand Surg. 2013;47:169-74.

15. Morris DE, Wu L, Zhao LL, et al. Acute and chronic animal models for excessive dermal scarring: quantitative studies. Plast Reconstr Surg. 1997; 100:674-81

16. Przybyt E, Krenning G, Brinker MG, Harmsen MC. Adipose stromal cells primed with hypoxia and inflammation enhance cardiomyocyte proliferation rate in vitro through STAT3 and Erk1/2. J Transl Med. 2013;11:39.

17. Kim D, Pertea G, Trapnell C, Pimentel H, Kelley R, Salzberg SL. TopHat2: accurate alignment of transcriptomes in the presence of insertions, deletions and gene fusions. Genome Biol. 2013;14:R36.

18. Trapnell C, Hendrickson DG, Sauvageau M, Goff L, Rinn JL, Pachter L. Differential analysis of gene regulation at transcript resolution with RNA-seq Nat Biotechnol. 2013;31:46-53.

19. Anders S, Pyl PT, Huber W. HTSeq--a Python framework to work with highthroughput sequencing data. Bioinformatics (Oxford, England). 2015;31:166-9.

20. Love MI, Huber W, Anders S. Moderated estimation of fold change and dispersion for RNA-seq data with DESeq2. Genome Biol. 2014;15:550.

21. Huang da, W., Sherman, B. T., Lempicki, R. A. Bioinformatics enrichment tools: paths toward the comprehensive functional analysis of large gene lists. Nucleic Acids Res 2009;37:1-13.

22. Huang da W., Sherman, B. T., Lempicki, R. A. Systematic and integrative analysis of large gene lists using DAVID bioinformatics resources. Nat Protoc 2009:4:44-57.

23. Xue $M$, Jackson CJ. Extracellular matrix reorganization during wound healing and its impact on abnormal scarring. Adv Wound Care. 2015;4:119-36.

24. Hinz B. Formation and function of the myofibroblast during tissue repair. J Invest Dermatol. 2007;127:526-37.

25. Uchida G, Yoshimura K, Kitano Y, Okazaki M, Harii K. Tretinoin reverses upregulation of matrix metalloproteinase-13 in human keloid-derived fibroblasts. Exp Dermatol. 2003;12(Suppl 2):35-42.

26. Desmouliere A, Geinoz A, Gabbiani F, Gabbiani G. Transforming growth factor-beta 1 induces alpha-smooth muscle actin expression in granulation tissue myofibroblasts and in quiescent and growing cultured fibroblasts. J Cell Biol. 1993;122:103-11.

27. Desmouliere A, Chaponnier C, Gabbiani G. Tissue repair, contraction, and the myofibroblast. Wound Repair Regeneration. 2005;13:7-12.

28. Yagmur C, Akaishi S, Ogawa R, Guneren E. Mechanical receptor-related mechanisms in scar management: a review and hypothesis. Plast Reconstr Surg. 2010;126:426-34.

29. Cubison TC, Pape SA, Parkhouse N. Evidence for the link between healing time and the development of hypertrophic scars (HTS) in paediatric burns due to scald injury. Burns. 2006;32:992-9.

30. Bloemen MC, van der Veer WM, Ulrich MM, van Zuijlen PP, Niessen FB, Middelkoop E. Prevention and curative management of hypertrophic scar formation. Burns. 2009:35:463-75.

31. Gabriel V. Hypertrophic scar. Phys Med Rehabil Clin N Am. 2011;22:301-10 vi.

32. Martinez FO, Helming L, Gordon S. Alternative activation of macrophages: an immunologic functional perspective. Annu Rev Immunol. 2009;27:451-83.

33. Pakyari M, Farrokhi A, Maharlooei MK, Ghahary A. Critical role of transforming growth factor Beta in different phases of wound healing. Adv Wound Care. 2013;2:215-24.

34. Spiekman M, Przybyt E, Plantinga JA, Gibbs S, van der Lei B, Harmsen MC. Adipose tissue-derived stromal cells inhibit TGF-beta1-induced differentiation of human dermal fibroblasts and keloid scar-derived fibroblasts in a paracrine fashion. Plast Reconstr Surg. 2014;134:699-712.

35. Gauglitz GG, Korting HC, Pavicic T, Ruzicka T, Jeschke MG. Hypertrophic scarring and keloids: pathomechanisms and current and emerging treatment strategies. Mol Med (Cambridge, Mass). 2011;17:113-25.

36. Thannickal VJ, Lee DY, White ES, et al. Myofibroblast differentiation by transforming growth factor-beta1 is dependent on cell adhesion and integrin signaling via focal adhesion kinase. J Biol Chem. 2003;278:12384-9. 
37. Cawston TE, Young DA. Proteinases involved in matrix turnover during cartilage and bone breakdown. Cell Tissue Res. 2010;339:221-35.

38. Zhang Q, Liu LN, Yong Q, Deng JC, Cao WG. Intralesional injection of adipose-derived stem cells reduces hypertrophic scarring in a rabbit ear model. Stem Cell Res Ther. 2015;6:145.

39. Madrigal M, Rao KS, Riordan NH. A review of therapeutic effects of mesenchymal stem cell secretions and induction of secretory modification by different culture methods. J Transl Med. 2014;12:260.

40. Gulotta LV, Kovacevic D, Ehteshami JR, Dagher E, Packer JD, Rodeo SA. Application of bone marrow-derived mesenchymal stem cells in a rotator cuff repair model. Am J Sports Med. 2009;37:2126-33.

41. Teng C, Zhou C, Xu D, Bi F. Combination of platelet-rich plasma and bone marrow mesenchymal stem cells enhances tendon-bone healing in a rabbit model of anterior cruciate ligament reconstruction. J Orthop Surg Res. 2016;1 1:96.

42. Phelps J, Sanati-Nezhad A, Ungrin M, Duncan NA, Sen A. Bioprocessing of mesenchymal stem cells and their derivatives: toward cell-free therapeutics. Stem Cells Int. 2018;2018:9415367.

43. L PK., Kandoi S., Misra R., S V., K R., Verma R. S. The mesenchymal stem cell secretome: a new paradigm towards cell-free therapeutic mode in regenerative medicine. Cytokine Growth Factor Rev 2019;46:1-9.

44. Lee DH, Ryu KJ, Kim JW, Kang KC, Choi YR. Bone marrow aspirate concentrate and platelet-rich plasma enhanced bone healing in distraction osteogenesis of the tibia. Clin Orthop Relat Res. 2014;472:3789-97.

45. Uder C, Bruckner S, Winkler S, Tautenhahn HM, Christ B. Mammalian MSC from selected species: features and applications. Cytometry Part A. 2018;93: 32-49.

\section{Publisher's Note}

Springer Nature remains neutral with regard to jurisdictional claims in published maps and institutional affiliations.

Ready to submit your research? Choose BMC and benefit from:

- fast, convenient online submission

- thorough peer review by experienced researchers in your field

- rapid publication on acceptance

- support for research data, including large and complex data types

- gold Open Access which fosters wider collaboration and increased citations

- maximum visibility for your research: over $100 \mathrm{M}$ website views per year

At $\mathrm{BMC}$, research is always in progress.

Learn more biomedcentral.com/submissions 